\title{
DEVELOPMENT AND IN VITRO EVALUATION OF PHYTOSOMES OF NARINGIN
}

\author{
JEEVANA JYOTHI B*, MARY RAGALATHA P
}

Department of Pharmaceutics, Institute of Pharmaceutical Technology, Sri Padmavati Mahila Visvavidyalayam (Women's University), Tirupati - 517 502, Andhra Pradesh, India. Email: jeevanajyothib8@gmail.com

Revised: 16 June 2019, Revised and Accepted: 27 July 2019

ABSTRACT

Objective: Phytosomes are novel herbal formulations meant for design of poorly soluble flavonoids of therapeutic potential. Naringin is a flavanone with poor oral absorption and bioavailability but possess antioxidant, anti-inflammatory, antiapoptotic, anti-ulcer, antiosteoporotic, and anticarcinogenic effects. Hence, the objective of the present work is the development of phytosomes of naringin to enhance its dissolution so that its therapeutic effects can be exploited.

Materials and Methods: Phytosomes containing $350 \mathrm{mg}$ of naringin were prepared by antisolvent precipitation method and rotary evaporation method using soya lecithin as main polymer. The prepared phytosomes were evaluated by entrapment efficiency, in vitro drug release studies, and drug-excipient interaction studies.

Results: Phytosomes made by rotary evaporation method evidenced higher dissolution values than phytosomes made by antisolvent precipitation. Formulation F7 containing $350 \mathrm{mg}$ of naringin and $1400 \mathrm{mg}$ of soya lecithin revealed the highest percentage release of $84.5 \pm 0.39 \%$ in $60 \mathrm{~min}$ and $99.7 \pm 0.24 \%$ in $120 \mathrm{~min}$. The percentage of drug entrapment efficiency values was satisfactory. Fourier-transform infrared spectroscopy spectra of pure naringin and naringin phytosomes revealed no interaction between the drug and polymers used for preparation.

Conclusion: Naringin phytosomes are produced successfully by the rotary evaporation method. Phytosomes made with 350 mg of naringin and $1400 \mathrm{mg}$ of soya lecithin by rotary evaporation method are spherical with a rough outer surface and optimum release characteristics of $84.5 \pm 0.39$ in $60 \mathrm{~min}$ to possess optimum bioavailability and $99.7 \%$ in $120 \mathrm{~min}$.

Keywords: Naringin, Flavonoid, Anticancer and Antioxidant, Poor bioavailability, Phytosomes, In vitro dissolution.

(C) 2019 The Authors. Published by Innovare Academic Sciences Pvt Ltd. This is an open access article under the CC BY license (http://creativecommons. org/licenses/by/4. 0/) DOI: http://dx.doi.org/10.22159/ajpcr.2019.v12i9.34798

\section{INTRODUCTION}

Naringin is a flavanone glycoside found in citrus fruits and mainly in grapefruit juice. It is used in Chinese herbal medicine as an active ingredient of Rhizoma Drynariae [1]. It is responsible for bitter taste of fruits. It is reported to possess important pharmacological activities such as antioxidant, anti-inflammatory, antiapoptotic, antiulcer, antiosteoporotic, and anticarcinogenic effects [2]. In humans, naringin is metabolized to aglycone naringenin by enzyme naringinase present in the gut. This happens in two steps; naringin is hydrolyzed by L-rhamnosidase activity of naringinase to rhamnose and prunin. The prunin formed is then hydrolyzed by the d-glucosidase activity of naringinase into naringenin and glucose.

Many of the bioactive constituents of phytomedicines are flavonoids and have poor oral absorption [3-5]. Naringin also has lower absorption and bioavailability due to two factors. These chief constituents are the number of ringed molecule and not too much small that it will absorb by the diffusion process. The second factor is that flavonoid molecule or chief constituent of polyphenols has poor solubility with lipids. These are limitations that inhibit their absorption through the biological membrane [6]. To overcome these limitations and to exploit the therapeutic benefits of flavonoids, herbal drug design and technological products such as phytosomes are recently introduced and developed. The term "phyto" means plant, while "some" means celllike. Phytosomes are also known as herbosomes [7]. It is a complex of natural active ingredient and a phospholipid mostly lecithin. These little cells protect plant extract or its active constituent from destruction by gastric secretions and gut bacteria due to gastroprotective property of phosphatidylcholine. It is claimed that phytosomes increase the absorption of conventional herbal extracts [8]. Some herbal compounds binding to phosphatidylcholine produce highly bio available form of herbal compounds. Hence, phytosomes improve the absorption, increasing bioavailability and enhancing delivery to the tissue [9-11].

Literature reveals that there are no formulations of naringin as phytosomes. Hence, the present research work is aimed at production of phytosomes of naringin with enhanced dissolution with optimum drug release characteristics so that the product will have optimum absorption and bioavailability.

\section{MATERIALS AND METHODS}

Naringin and soya lecithin were purchased from HiMedia Pvt. Ltd. Methanol and dichloromethane were purchased from Sigma Aldrich Pvt. Ltd. n-Hexane was from SDL laboratories and all other chemicals used in the study were of analytical grade.

\section{Preparation of phytosomes of naringin}

Phytosomes of naringin were prepared by antisolvent precipitation method and rotary evaporation method.

\section{Antisolvent precipitation method}

Various ratios of naringin and soya lecithin were used to prepare naringin phytosomes by antisolvent precipitation method. Formulations with which discrete particles are not obtained are discarded, and three formulations (F1 to F3) successful in producing discrete particles are given in Table 1. During preparation, naringin and soya lecithin were placed in $100 \mathrm{ml}$ round-bottomed flask and refluxed with dichloromethane for $2 \mathrm{~h}$. When the solution was concentrated to 5-10 ml, $30 \mathrm{ml}$ of $\mathrm{n}$-hexane was added to get the complex as a precipitate. The precipitate was collected and placed in a desiccator for $24 \mathrm{~h}$. The dried precipitate was crushed in mortar and sieved through \#100 mesh. Powdered complex was placed in amber-colored glass bottle and was stored at room temperature. 
Table 1: Composition of naringin phytosomes made by antisolvent precipitation method

\begin{tabular}{lllll}
\hline Formulation code & Drug $(\mathbf{m g})$ & Soya lecithin $\mathbf{( m g )}$ & Dichloromethane $(\mathbf{m l})$ & $\mathbf{n}$-hexane $(\mathbf{m l})$ \\
\hline F1 & 350 & 350 & 30 & 10 \\
F2 & 350 & 700 & 30 & 10 \\
F3 & 350 & 1050 & 30 & 10 \\
\hline
\end{tabular}

Table 2: Composition of naringin phytosomes made by rotary evaporation method

\begin{tabular}{llllll}
\hline Formulation code & Drug $(\mathbf{m g})$ & Soya lecithin $(\mathbf{m g})$ & Methanol $(\mathbf{m l})$ & Dichloromethane $(\mathbf{m l})$ & pH 7.4 phosphate buffer $(\mathbf{m l})$ \\
\hline F4 & 350 & 350 & 30 & 20 & 25 \\
F5 & 350 & 700 & 30 & 20 & 25 \\
F6 & 350 & 1050 & 30 & 20 & 25 \\
F7 & 350 & 1400 & 30 & 20 & 25 \\
\hline
\end{tabular}

Table 3: Entrapment efficiency of naringin phytosomes

\begin{tabular}{ll}
\hline Formulation code & \% EE \\
\hline F1 & $72.34 \pm 0.09$ \\
F2 & $81.17 \pm 0.21$ \\
F3 & $83.64 \pm 0.19$ \\
F4 & $91.91 \pm 0.44$ \\
F5 & $91.31 \pm 0.14$ \\
F6 & $90.01 \pm 0.21$ \\
F7 & $92.91 \pm 0.11$ \\
\hline${ }^{*} \mathrm{n}=3+$ S.D &
\end{tabular}

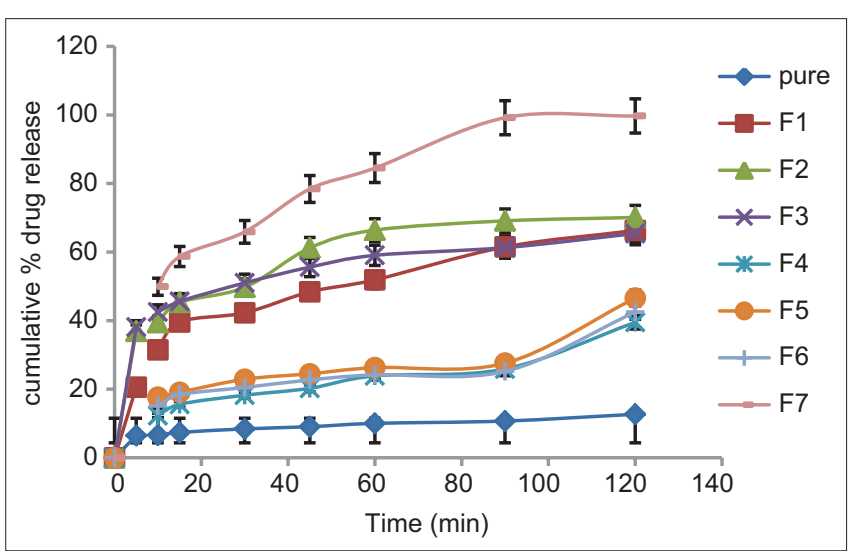

Fig. 1: Dissolution curves of naringin and naringin phytosomes F1 toF

Rotary evaporation method

Five trials of naringin formulations were tried rotary evaporation method and their composition is given in Table 2. Various ratios of naringin to soya lecithin, namely $1: 1,1: 2,1: 3,1: 4$, and 1:5, were taken in the compositions, as shown in Table 1.

Accurately weighed quantity of naringin (drug) was dissolved in methanol and soya lecithin was dissolved in dichloromethane. The mixture of two solutions was transferred to round-bottomed flask and refluxed for $2 \mathrm{~h}\left(60^{\circ} \mathrm{C}\right)$. At this stage, the solution was obtained in a clear yellow color solution. The mixture was taken in RBF and evaporated in a rotary evaporator at $60^{\circ} \mathrm{C}$ until all solvents evaporated perfectly and a thin film was produced. The thin film was hydrated with phosphate buffer 7.4, and the suspension was filtered, collected, and dried under vacuum and was stored in amber color bottles.

\section{In vitro evaluation of prepared phytosomes}

Drug entrapment efficiency

The percentage of drug entrapment efficiency was determined for all the prepared naringin phytosomes (F1 to F7). $100 \mathrm{mg}$ of the product was weighed and transferred into $100 \mathrm{ml}$ volumetric flask containing
$100 \mathrm{ml}$ of phosphate buffer (pH 6.8) and was kept it aside. On the next day, the volumetric flask was kept for continues stirring for $2 \mathrm{~h}$ at $35^{\circ} \mathrm{C}$ to complete release of drug from the formulation. Then, the solution was filtered, and $1 \mathrm{ml}$ sample was diluted to $10 \mathrm{ml}$ and analyzed for the drug entrapment efficiency using ultra violet (UV) spectrophotometer at $262 \mathrm{~nm}$. Drug entrapment was calculated using the formula.

Percentage drug entrapment efficiency $=\frac{\text { Actual drug content }}{\text { Theoretical content }} \times 100$

In vitro drug release studies

In vitro dissolution studies were carried for pure naringin and also for prepared naringin phytosomes (F1 to F7) using USP Type II dissolution test apparatus (Labindia DS 8000, India). $900 \mathrm{ml}$ of pH 7.4 phosphate buffer maintained at $37 \pm 0.5^{\circ} \mathrm{C}$ temperature and $100 \mathrm{rpm}$ was used as a dissolution medium. Phytosomes equivalent to contain $350 \mathrm{mg}$ of naringin was placed in the medium. At predetermined time intervals, $5 \mathrm{ml}$ of each sample was withdrawn at regular time intervals $(0,5,10$, $20,30,45,60,90$, and $120 \mathrm{~min}$ ) and was replaced by an equal volume of fresh medium. The samples were filtered and diluted and analyzed on UV spectrophotometer (Shimadzu, UV 1700) at $283 \mathrm{~nm}$.

\section{Assessment of drug release kinetics}

Rate and mechanism of drug release from matrix tablets was assessed by fitting the data into zero-order kinetics $\left[\mathrm{Q}=\mathrm{K}_{\mathrm{o}} \mathrm{t}\right]$, first-order kinetics $\left[\log \mathrm{Q}=\log \mathrm{Q}_{0}+\mathrm{K}_{1} \mathrm{t} / 2.303\right]$, Higuchi's model $[\mathrm{Q}=\mathrm{KH} \mathrm{t} / 2 \mathrm{2}]$, and KorsmeyerPeppas model $\left[\mathrm{F}=(\mathrm{Mt} / \mathrm{M})=\mathrm{K} \mathrm{t}_{\mathrm{n}}\right]$

Phase-contrast microscopy of naringin phytosomes

For determination of the shape of of naringin phytosomes, small amount of phytosomes are kept on the glass slide and spread over the slide. Then, the microscopy was adjusted to $\times 10$ and focusing on the slide by adjusting the stage micrometer. Then, the shape of the phytosomes was observed on the screen and the photograph was taken.

Fourier-transform infrared spectroscopy (FTIR) of naringin phytosomes Drug excipient interaction between the naringin and the ingredients used for design of phytosomes was investigated by FTIR by potassium bromide pressed pellet technique (Bruker, Japan). The FTIR spectrum was obtained for pure naringin and for phytosomes of naringenin transmittance mode in the range of $400-4000 \mathrm{~cm}^{-1}$.

\section{RESULTS AND DISCUSSION}

Phytosomes of naringin were produced successfully by both the selected methods such as antisolvent precipitation method (F1, F2, and F3) and rotary evaporation method (F4 to F7). All formulations (F1 to F7) were subjected to in vitro evaluation by \%entrapment efficiency, drug release studies, and release kinetics. Promising formulation with enhanced dissolution rate was investigated by phase-contrast microscopy to identify appearance and by drug-excipient interaction studies by FTIR. 
Table 4: \% Drug dissolved versus time values of pure naringin and naringin phytosomes (F1 to F7)

\begin{tabular}{|c|c|c|c|c|c|c|c|c|}
\hline Time (min) & Pure drug & F1 & F2 & F3 & F4 & F5 & F6 & F7 \\
\hline 5 & $6.48 \pm 0.21$ & $20.6 \pm 0.18$ & $36.9 \pm 0.25$ & $38.1 \pm 0.34$ & $7.7 \pm 0.22$ & $10.35 \pm 0.51$ & $9.35 \pm 0.21$ & $35.5 \pm 0.21$ \\
\hline 10 & $6.61 \pm 0.12$ & $31.5 \pm 0.35$ & $39.4 \pm 0.34$ & $42.5 \pm 0.19$ & $12.3 \pm 0.43$ & $17.7 \pm 0.42$ & $15.1 \pm 0.63$ & $49.9 \pm 0.12$ \\
\hline 15 & $7.38 \pm 0.35$ & $39.6 \pm 0.24$ & $45.3 \pm 0.16$ & $45.6 \pm 0.21$ & $15.6 \pm 0.32$ & $19.1 \pm 0.42$ & $18.4 \pm 0.11$ & $58.7 \pm 0.35$ \\
\hline 30 & $8.43 \pm 0.18$ & $42.3 \pm 0.48$ & $49.7 \pm 0.18$ & $51.01 \pm 0.24$ & $18.25 \pm 0.75$ & $22.9 \pm 0.35$ & $20.5 \pm 0.44$ & $65.9 \pm 0.58$ \\
\hline 45 & $9.06 \pm 0.36$ & $48.4 \pm 0.15$ & $61.2 \pm 0.17$ & $55.6 \pm 0.15$ & $20.27 \pm 0.42$ & $24.5 \pm 0.28$ & $22.7 \pm 0.43$ & $78.4 \pm 0.35$ \\
\hline 60 & $10.03 \pm 0.55$ & $51.9 \pm 0.14$ & $66.4 \pm 0.27$ & $59.05 \pm 0.26$ & $23.9 \pm 0.51$ & $26.3 \pm 0.15$ & $24.2 \pm 0.41$ & $84.5 \pm 0.39$ \\
\hline 90 & $10.71 \pm 0.24$ & $61.5 \pm 0.19$ & $69.1 \pm 0.22$ & $61.3 \pm 0.34$ & $25.9 \pm 0.30$ & $27.6 \pm 0.89$ & $25.2 \pm 0.62$ & $99.2 \pm 0.22$ \\
\hline
\end{tabular}

Table 5: Data of drug release kinetics plots

\begin{tabular}{|c|c|c|c|c|c|c|c|}
\hline Time (min) & $\begin{array}{l}\text { \% Cumulative } \\
\text { drug release }\end{array}$ & $\begin{array}{l}\text { Log\% Cumulative } \\
\text { drug release }\end{array}$ & $\begin{array}{l}\% \text { Drug } \\
\text { retained }\end{array}$ & $\begin{array}{l}\text { Log \% drug } \\
\text { retained }\end{array}$ & $\log T$ & $\sqrt{\mathrm{T}}$ & $\sqrt[3]{T}$ \\
\hline 0 & 0 & 0 & 100 & 2 & 0 & 0 & 0 \\
\hline 5 & 35.5 & 1.5502 & 64.5 & 1.809 & 0.698 & 2.23 & 1.709 \\
\hline 10 & 49.9 & 1.6981 & 50.1 & 1.699 & 1 & 3.16 & 2.154 \\
\hline 15 & 58.7 & 1.7686 & 41.3 & 1.615 & 1.176 & 3.87 & 2.466 \\
\hline 30 & 65.9 & 1.8188 & 34.1 & 1.532 & 1.477 & 5.47 & 3.107 \\
\hline 45 & 78.4 & 1.8943 & 21.6 & 1.334 & 1.653 & 6.70 & 3.556 \\
\hline 90 & 99.2 & 1.9965 & 0.8 & 0.096 & 1.954 & 9.48 & 4.481 \\
\hline 120 & 99.7 & 1.9986 & 0.3 & 0.522 & 2.079 & 10.95 & 4.932 \\
\hline
\end{tabular}

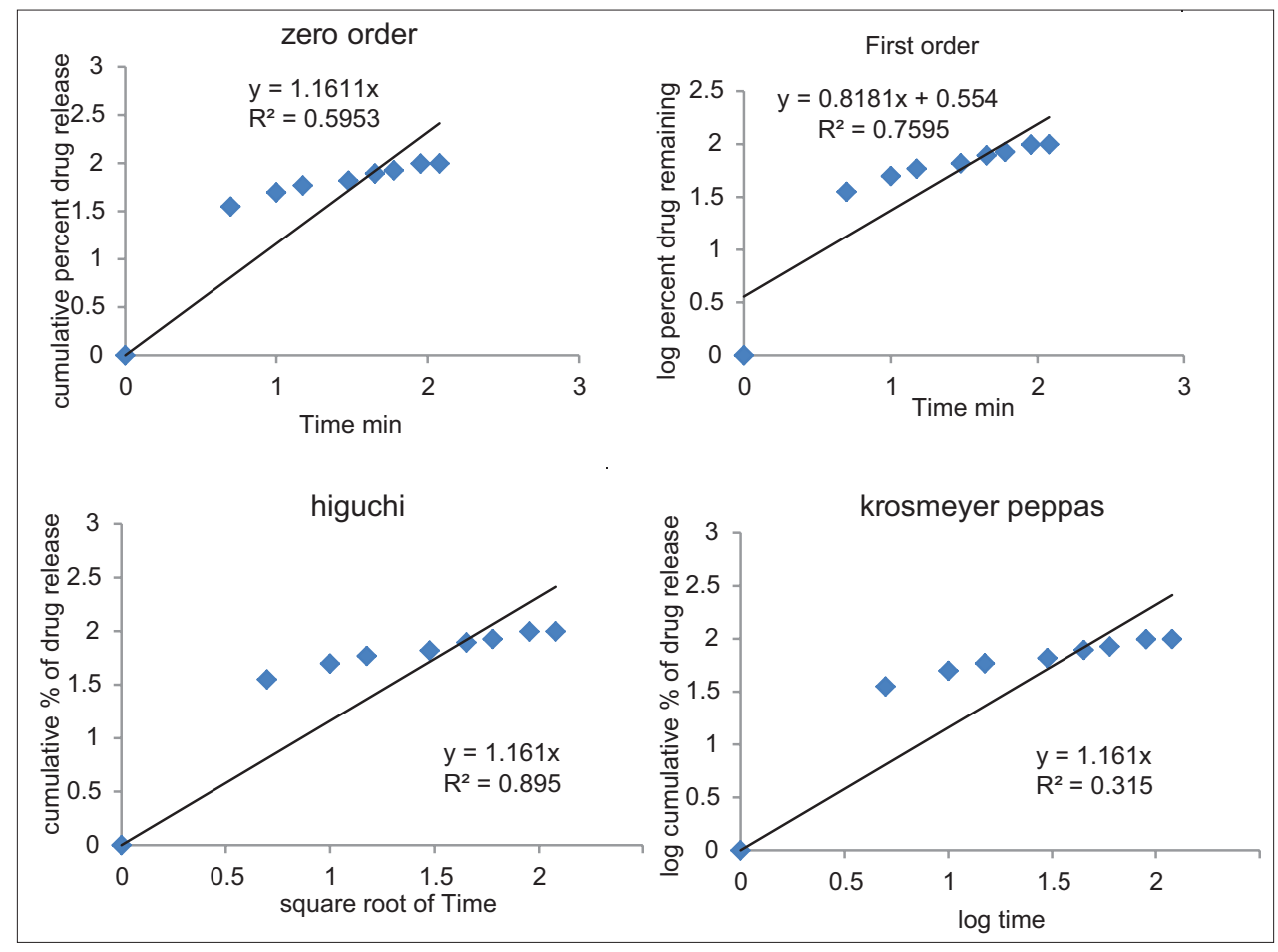

Fig. 2: Kinetic plots of druu release from naringinphytosomes F7

Drug entrapment efficiency

Drug entrapment efficiency values of prepared phytosomes of naringin, F1 to F7, are shown in Table 3 and are found satisfactory in all the formulations. However there better value with formulations made by rotary evaporation method F4 to F7.

\section{In vitro drug release studies}

The results of in vitro drug release studies of pure naringin and phytosomes of naringin made by antisolvent precipitation method (F1 to F3) and rotary evaporation method (F4 to F7) are shown in Fig. 1 and Table 4. Pure drug evidenced only $12.69 \%$ in 120 min, indicating poor solubility of naringin. However, there is enhancement of drug dissolution when it is made as phytosomes. Phytosomes made by rotary evaporation method (F4 to F7) evidenced higher dissolution values than phytosomes made by antisolvent precipitation (F1 to F3). Formulation F7 exhibited the highest percentage as $84.5 \pm 0.39$ in $60 \mathrm{~min}$ and $99.7 \pm 0.24 \%$ in $120 \mathrm{~min}$. Hence, this was considered as promising formulation and was further investigated for drug-excipient interaction studies.

Drug release kinetics of naringin phytosomes

Drug release kinetics of promising formulation (F7) was assessed by plotting zero-order, first-order, Higuchi, and Korsmeyer-Peppas (k-p) plots. The corresponding data and the plots are shown in Table 5 and 
Table 6: Correlation coefficient, $\mathbf{R}^{2}$ values

\begin{tabular}{lll}
\hline Plot & $\mathbf{R}^{2}$ value & \\
\hline Zero order & 0.595 & \\
First order & 0.759 & \\
Higuchi plot & 0.895 & $1.161(\mathrm{n})$ \\
\hline Korsmeyer-peppas plot & 0.315 & 15 \\
\hline
\end{tabular}

Table 7: Fourier-transform infrared spectroscopy absorption of naringin and drug-loaded phytosomes

\begin{tabular}{lll}
\hline $\begin{array}{l}\text { Functional groups } \\
\text { of naringin }\end{array}$ & $\begin{array}{l}\text { Infrared absorption } \\
\text { of naringin }\end{array}$ & $\begin{array}{l}\text { Infrared absorption } \\
\text { of naringin } \\
\text { phytosomes } \mathbf{F 7}\end{array}$ \\
\hline $\begin{array}{l}\text { OH stretching } \\
(3400-3300)\end{array}$ & $3400 \mathrm{~cm}^{-1}$ & $3334 \mathrm{~cm}^{-1}$ \\
$\begin{array}{l}\text { C-H stretching } \\
(2890-2970)\end{array}$ & $2900 \mathrm{~cm}^{-1}$ & $2924 \mathrm{~cm}^{-1}$ \\
$\begin{array}{l}\text { C=0 stretching } \\
(1646)\end{array}$ & $1643 \mathrm{~cm}^{-1}$ & $1638 \mathrm{~cm}^{-1}$ \\
C=C stretching & $1518 \mathrm{~cm}^{-1}$ & $1518 \mathrm{~cm}^{-1}$ \\
$(1517)$ & & \\
\hline
\end{tabular}

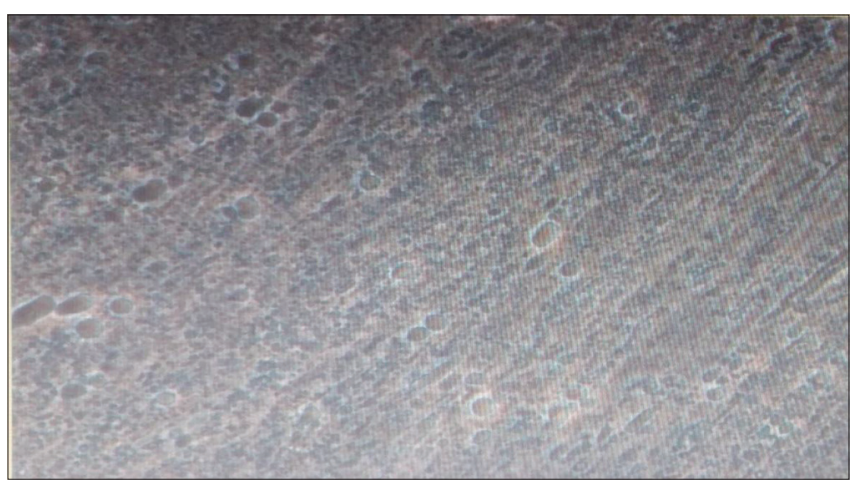

Fig. 3: Shape of phytosome of naringin
Fig. 2. Correlation coefficient $\left(\mathrm{R}^{2}\right)$ values of the plots are summarized in Table 6. More linear plots in case of first order indicate that the drug release is dose dependent and in case of Higuchi plot indicate that the mechanism of release is by diffusion. The $n=1.161$ in Korsmeyerpeppas plot $(>0.45$ and $<2)$ indicates non-Fickian of drug release.

\section{Phase-contrast microscopy of naringin phytosomes}

The photographs of the optimized formulation (F7) taken in phasecontrast microscopy are depicted in Fig. 3. The phase-contrast microscopy photographs revealed that the phytosomes are discrete and spherical in shape with a rough outer surface morphology which could be because of the surface association of the drug with the polymer.

\section{FTIR studies of naringin phytosomes}

Infrared (IR) spectra of pure naringin and promising formulation of naringin phytosomes (F7) are shown in Figs. 4 and 5, respectively. Comparative data are shown in Table 7. IR spectrum of naringin showed its characteristic peaks at $3400 \mathrm{~cm}^{-1}$ due to $\mathrm{O}-\mathrm{H}$ stretching, amide $\mathrm{C}=0$ stretch at $1643 \mathrm{~cm}^{-1}$, aromatic $\mathrm{C}=\mathrm{C}$ stretching at $1518 \mathrm{~cm}^{-1}$, and C-H stretching at $2900 \mathrm{~cm}^{-1}$. Phytosome formulation, F7, revealed $\mathrm{O}-\mathrm{H}$ stretching at $3334 \mathrm{~cm}^{-1}$, amide $\mathrm{C}=0$ stretch at $1638 \mathrm{~cm}^{-1}$, aromatic $\mathrm{C}=\mathrm{C}$ stretching at $1518 \mathrm{~cm}^{-1}$, and $\mathrm{C}-\mathrm{H}$ stretching at $2924 \mathrm{~cm}^{-1}$. This slight shift of these peaks indicates no interaction between the drug and polymers.

\section{CONCLUSION}

Naringin phytosomes are produced successfully by antisolvent precipitation method and rotary evaporation method. Phytosomes made with $350 \mathrm{mg}$ of naringin and $1400 \mathrm{mg}$ of soya lecithin by rotary evaporation method are spherical with a rough outer surface and optimum release characteristics of $84.5 \pm 0.39$ in 60 min to possess optimum bioavailability.

\section{ACKNOWLEDGMENTS}

The author acknowledges the gratitude to DST-CURIE facilities of Sri Padmavati Mahila Visvavidyalayam, Tirupati, for providing instrumentation such as FTIR, phase transition microscope, and UV-visible spectrophotometer required to carry out this work.

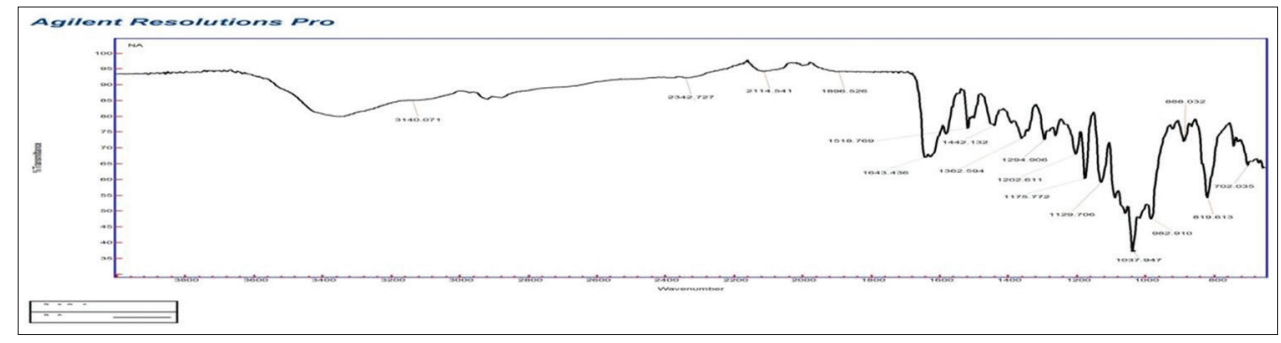

Fig. 4: Fourier-transform infrared spectroscopy spectrum of pure naringin

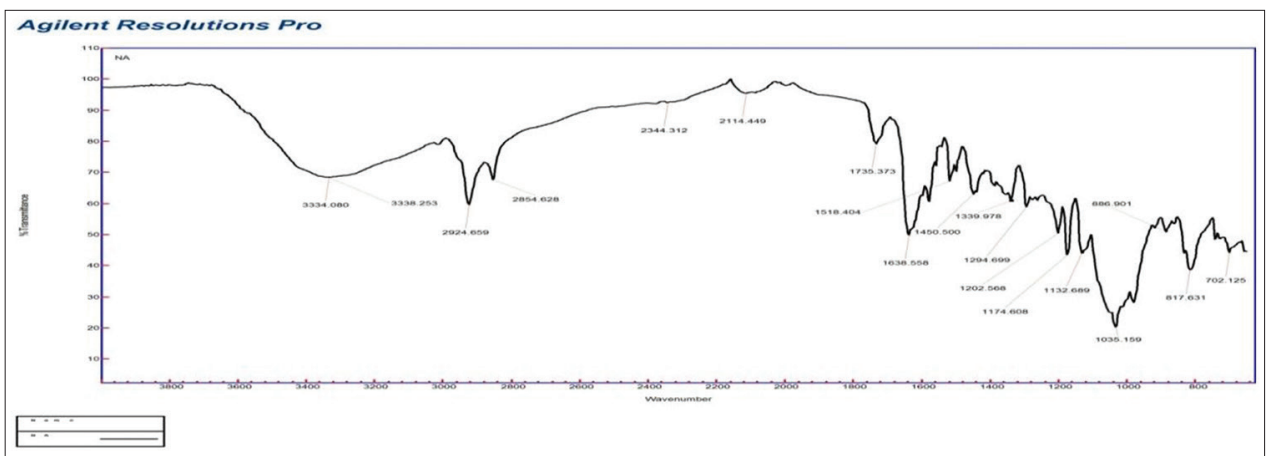

Fig. 5: Fourier-transform infrared spectroscopy spectrum of phytosome formulation F7 


\section{AUTHOR'S CONTRIBUTIONS}

Jeevana Jyothi B has designed the plan of the present research work and is responsible for the outcome of this novel work as well as preparation of the manuscript. Ragalatha has done the experiments involved in the research.

\section{REFERENCES}

1. Chen R, Qi QL, Wang MT, Li QY. Therapeutic potential of naringin: An overview. Pharm Biol 2016;54:3203-10.

2. Semalty A, Semalty M, Singh D, Ravat MS. Preparation and characterization of phospholipid complexes of naringenin for effective drug delivery. J Incl Phenom 2010;67:253-60.

3. Jain N, Gupta BP, Thakur N, Jain R, Banweer J, Jain DK, et al. Phytosome: A novel drug delivery system for herbal medicines. Int $\mathrm{J}$ Pharm Sci Drug Res 2010;2:224-8.

4. Patel J, Patel R, Khambojia K, Patel N. An overview of phytosomes as an advanced herbal drug delivery system. Asian J Pharm Sci 2009;4:363-71.
5. Panduraju T, Reddy MS, Veerareddy PR. Phytosomes; A novel phyto-phospholipid carriers for herbal drug delivery. Int Res J Pharm 2011:2:28-33.

6. Saha S, Sarma A, Saikia P, Chakraborty T. Phytosomes: A brief overview. Sch Acad J Pharm 2013;2:12-20.

7. Agrawal VK, Gupta A, Chaturvedi S. Improvement in bioavailability of class-III drug; phytolipid delivery system. Int J Pharm Pharm Sci 2012;4:37-42.

8. Swati R, Sapna M. Phytosomal drug delivery systems. Int J Res Dev Pharm Life Sci 2012;1:143-50.

9. Khan J, Alexander A, Ajazuddin, Saraf S, Saraf S. Recent advances and future prospects of phyto-phospholipid complexation technique for improving pharmacokinetic profile of plant actives. J Control Release 2013;168:50-60.

10. Avasti R, Kulkarni GT, Pawar V. Phytosomes, an approach to incrse the bioavailability of plant products. Int J Pharm Pharm Sci 2011;3:1-3.

11. Ittadwar PA, Puranik PK. Umbelliferonephytosomes: Development and optimization using experimental design approach and evaluation of photoprotective and antioxidant activity. Int J Pharm Pharm Sci 2016;9:218-28. 